\title{
In Vitro Growth, Antrum Formation and Metaphase II Development of Preantral Follicles in Sheep with Estradiol-17ß Supplementation at Different Time Points
}

\author{
L. S. S. Varaprasad Reddy ${ }^{1}$, B. R. Naik ${ }^{1}$, A. V. N. Sivakumar ${ }^{1 *}$, \\ B. Punyakumari ${ }^{2}$ and J. Suresh ${ }^{3}$ \\ ${ }^{1}$ Dept. of Veterinary Physiology, ${ }^{2}$ Dept. of AGB, ${ }^{3}$ CCVEC, Sri Venkateswara Veterinary \\ University, Tirupati - 517502, Andhra Pradesh, India \\ *Corresponding author
}

Keywords

In vitro, Preantral follicles, Estradiol$17 \beta$, Sheep

Article Info

Accepted:

15 December 2019

Available Online:

20 January 2020
A B S T R A C T

The present study was conducted to investigate the influence of sequential addition of estradiol-17 $\beta$ on in vitro development of preantral follicles (PFs') in sheep. Preantral follicles isolated from the ovarian cortical slices using micro dissection method were cultured for six days in Bicarbonate buffered Tissue culture medium 199B (TCM 199B) or in a standard culture medium supplemented with Estradiol-17 $\beta(5 \mathrm{ng} / \mathrm{ml})$ at different points during the culture period. COCs isolated from the follicles at the end of six day culture in different treatments were subjected to in vitro maturation for additional 24h. Supplementation of estradiol-17 $\beta$ during first two days of the culture supported better average increase in diameter and proportion of PFs' exhibiting antrum formation at the end of six day culture. Further the oocytes in COCs isolated at the end of culture in these treatments and subsequently subjected to in vitro maturation (IVM) for $24 \mathrm{hr}$ developed at a higher frequency to MII (metaphase II) stage. Supplementation of Estradiol-17 $\beta$ to TCM 199B culture medium in early stages followed by standard medium alone in later stages supports better development of PFs' in vitro. Following supplementation with estradiol-17 $\beta$ for the first two days culture of PFs' in standard medium appears to be advantageous for the development of preantral follicles in vitro. 


\section{Introduction}

Mammalian females are born with thousands to lakhs of oocytes in the primordial follicles in the ovaries. But less than one percent of the primordial follicles grow to become Graafian follicles and ovulate. The remaining primordial follicles degenerate through a process known as atresia. This fact has stimulated great interest in the development of a culture system that might be able to maintain follicular growth and avoid this loss of follicles (Araujo et al., 2014). Accordingly several laboratories world over have been developing techniques to harvest, culture and produce embryos from the oocytes in preantral follicles (PFs') in the ovaries of different species of laboratory and domestic animals. Recent innovations to augment reproductive efficiency of female mammals such as superovulation, embryo transfer, in vitro embryo production, somatic cell cloning etc. are all oocyte intensive technologies. Such techniques could provide access to a new source of female germplasm and represent a huge opportunity even with a low over all success rate of just one percent since it would be possible to produce one thousand or more embryos from both the ovaries of a slaughtered female mammal.

In addition to the potential for greater use of the female germplasm. Estradiol is a hormone synthesized by aromatase, present in the granulosa cells and its production depends on gonadotrophin secretion (Nelson and Bulun, 2001). It is indeed known that estradiol can act as an inhibitor of the apoptosis induced by oxidative stress in luteal and follicular cells (Murdoch, 1998). Estradiol may act to support granulosa cell differentiation, after which these cells become receptive to gonadotrophin. Estradiol typically acts through specific intracellular receptors, ER $\alpha$ and ER $\beta$, which are expressed in granulosa cells of preantral and antral follicles (Tomic et al. 2007). The present study was conducted to study the influence of sequential addition of estradiol$17 \beta$ on in vitro development of preantral follicles (PFs') in sheep, and to study the influence of estradiol-17 $\beta$ on development of preantral follicles (PFs') in sheep at different time intervals.

\section{Materials and Methods}

Sheep ovaries were collected irrespective of age, body condition and season at local slaughter house. Preantral follicles were isolated as per the methods standardized in the laboratory. In brief the ovarian cortex was cut into thin slices from which intact preantral follicles in the size range of $250-400 \mu \mathrm{m}$ were mechanically isolated without damaging to the basement membrane by microdissection method under stereo zoom microscope. Total 9 treatment groups (T1 to T9) as represented in Table.1. Standard medium) was prepared by supplementing Bicarbonate buffered tissue culture medium 199 (TCM 199B) with Gentamycin (50 $\mu \mathrm{l} / \mathrm{ml}) ; \mathrm{T}_{4}(100 \mu \mathrm{l} / \mathrm{ml}) ; \mathrm{FSH}(175 \mu \mathrm{l} / \mathrm{ml})$; IGF-1 $(200 \mu \mathrm{l} / \mathrm{ml})$; GH $(200 \mu \mathrm{l} / \mathrm{ml})$ which supported the best development in vitro of sheep PFs earlier (Arunakumari et al., 2010). Preantral follicles were cultured in micro drops of TCM 199B / Standard medium supplemented variously with estradiol-17 $\beta$ (5 ng/ml) for a period of six days. Follicles in culture were morphologically evaluated every $24 \mathrm{~h}$ for the proportions exhibiting growth, averege increase in diameter and proportion of PFs' exhibiting antrum formation. Cumulus Oocyte complexes (COCs) were collected at the end of six day culture and subjected to additional $24 \mathrm{~h}$ of in vitro maturation. Meiotic maturation to MII stage was assessed by staining the oocytes with Hoechst 33342 medium. Statistical analysis of the data was analyzed by one way ANOVA by using SPSS 20 software. Duncan's multiple range test (DMRT) and percentile deviation tests were applied to 
identify the significance of difference among different treatment groups.

\section{Results and Discussion}

The present study demonstrated the importance of estradiol for growth, activation and maturation of sheep preantral follicles. In our present experiment supplementation of estradiol $17 \beta$ did not showed the significance difference in proportion of PFs' exhibited growth, increase in diameter, antrum formation and in vitro maturation of oocytes to M-II stage (Table. 2).However, increase in diameter $\left(28.99 \pm 3.37^{\mathrm{a}}\right)$, antrum formation $\left(48.33 \pm 4.61^{\mathrm{a}}\right)$ and in vitro maturation of oocytes to M-II stage $\left(14.99 \pm 6.30^{\mathrm{a}}\right)$ were better in treatment where estradiol $17 \beta$ supplemented first two days followed by culture in standard medium.

Although there are few studies on the influence of estradiol on growth, antrum formation and maturation, it is known that estradiol can act as an inhibitor of the apoptosis in luteal and follicular cells (Murdoch, 1998). Lima-Verde et al. (2010) observed supplementation of $1 \mathrm{pg} / \mathrm{ml}$ estradiol with $50 \mathrm{ng} / \mathrm{ml}$ of FSH promoted caprine primordial follicle activation and further in vitro follicular growth. Zheng et al. (2003) observed oocyte developmental capacity during IVM in rhesus monkey was stimulated by supplementation of $17 \beta$ estradiol. Similar results were reported by Tasaki et al. (2013), they observed highest rate of antrum formation with supplementation of culture medium with $1 \mu \mathrm{g} / \mathrm{ml}$ estradiol and no antrum formation without estradiol in PFs' of pig. Proper $17 \beta$ estradiol levels promote follicle growth and inhibit follicle atresia. $17 \beta$ estradiol was thought to exert its role in promoting folliculogenesis by favoring granulosa cell proliferation and inhibiting apoptosis signals. The result of our study, in which supplementation of estradiol-17 $\beta$ (5 $\mathrm{ng} / \mathrm{ml}$ ) to TCM 199B culture medium in early stages followed by standard medium alone in later stages supports better development of PFs' in vitro. Endo et al. (2013) observed supplementation of the culture medium with estradiol improved the cavity formation, and the highest ratio of antrum cavity formation was obtained with the medium containing 10 $\mu \mathrm{g} / \mathrm{ml}$ estradiol-17 $\beta$. Various studies have reported that $17 \beta$ estradiol favors cell proliferation and survival by activating the transcription of factors required for progression through the cell cycle, while repressing others that cause cell cycle arrest and apoptosis (Craig et al., 2014). In the human, IVM of oocytes in media containing $17 \beta$-estradiol $\left(\mathrm{E}_{2}\right)$ supports the cytoplasmic maturation which is necessary for fertilization and early embryonic development (Tesarik and Mendoza, 1995). This is in agreement with findings of Ali and Sirard (2002), where oocytes matured in the presence of higher concentration of E2 $(1000 \mathrm{ng} / \mathrm{ml})$ improved their developmental rate to the morula and blastocyst stages. Therefore, E2 may play an important role in the process of follicular development and ovulation. However, the developmental potential of matured bovine oocytes to the morula and blastocyst stages was not improved by the presence of lower concentrations of $\mathrm{E}_{2}(10$ or $100 \mathrm{ng} / \mathrm{ml})$ (Ali and Sirard, 2002).

However, Li et al., (2004) observed the inhibitory effects of $17 \beta$-estradiol on nuclear and cytoplasmic maturation of cumulus enclosed pig oocytes. These inhibitory effects were prevented when the medium was supplemented with $17 \beta$-estradiol together with its antagonist. Similarly, Beker et al., (2002) also observed that estradiol negatively affects bovine oocyte nuclear maturation as reflected by decrease of the proportion of MII oocytes and by the increase of occurrence of nuclear aberrations. 
Table.1 Experimental design for time specific supplementation of Estradiol-17ß (5 ng/ml) in vitro culture of preantral follicles in Sheep

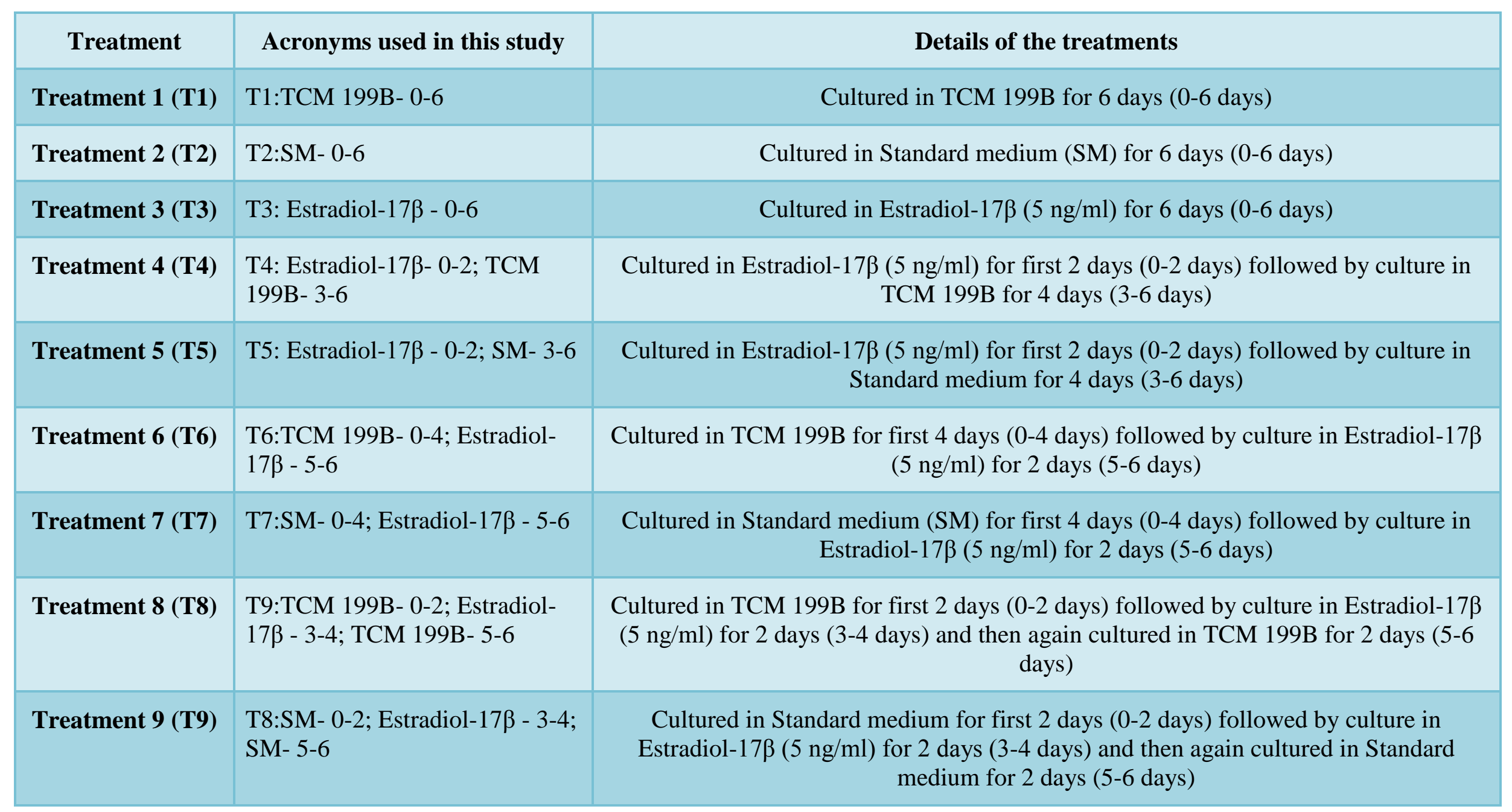


Table.2 Influence of supplementation of Estradiol-17ß (5 ng/ml) at different time points during in vitro culture of Sheep preantral follicles (PFs')

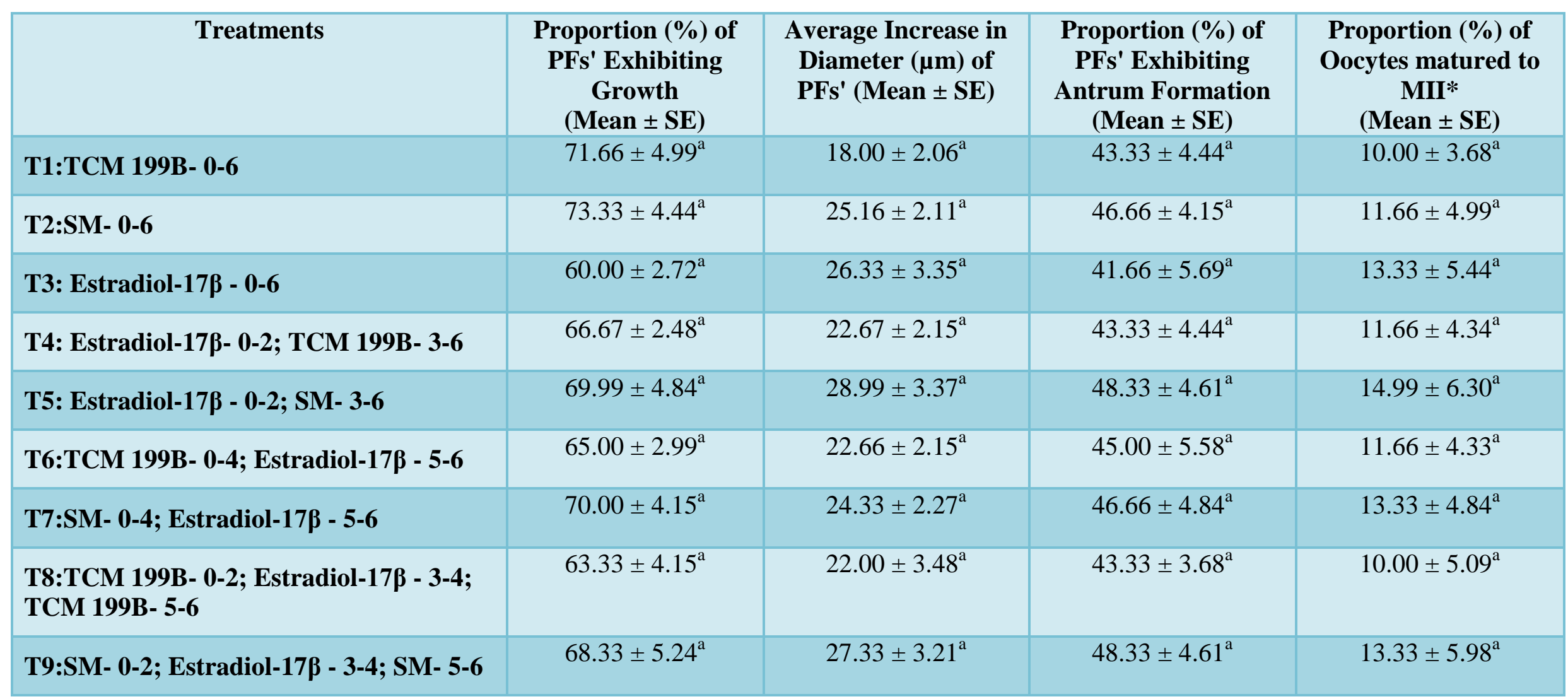

Figures with different superscripts within a column are significantly different $(\mathrm{P} \leq 0.05)$

* Oocytes in COCs isolated from six day cultured follicles in different treatments and subjected to IVM for additional 24hrs 

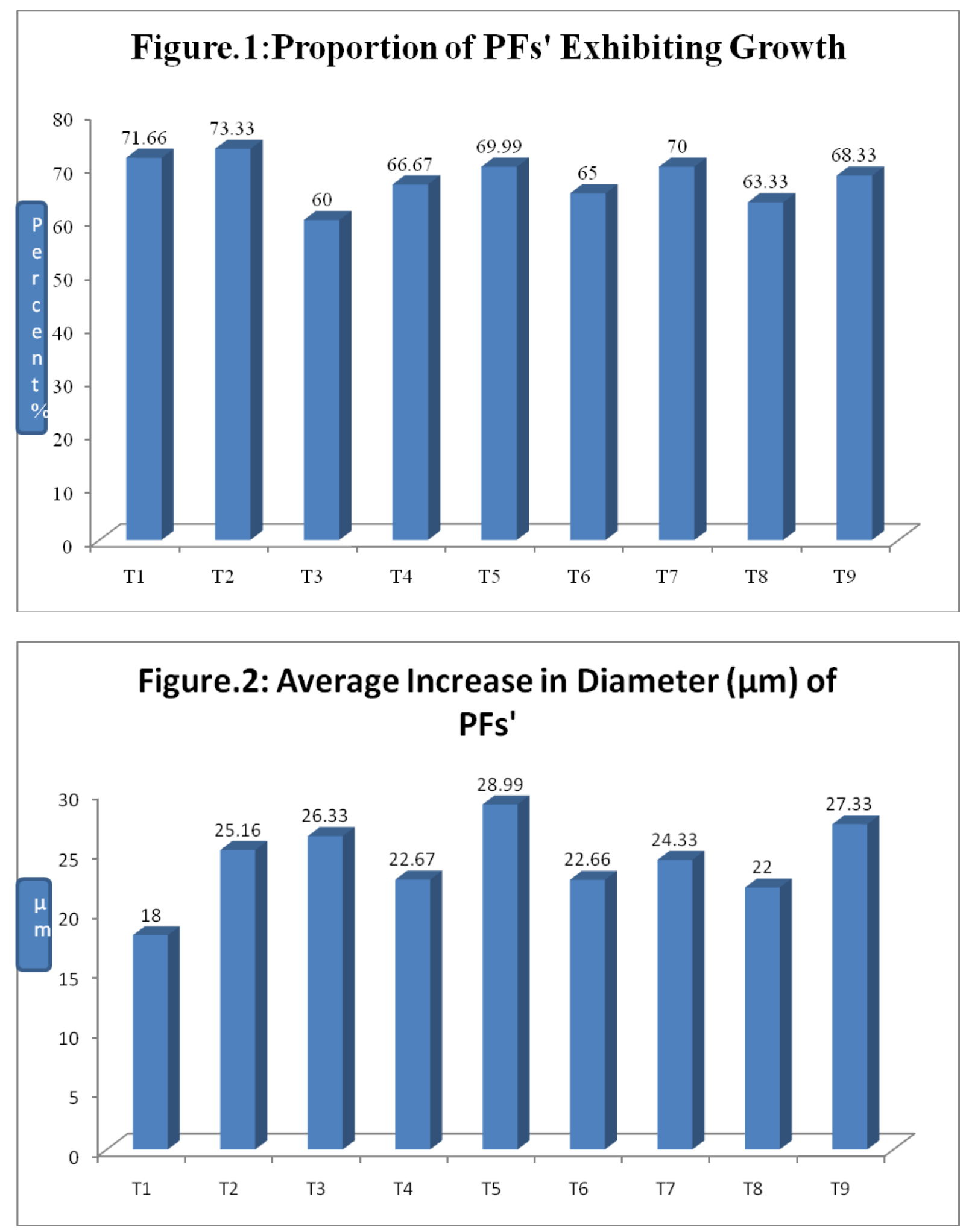


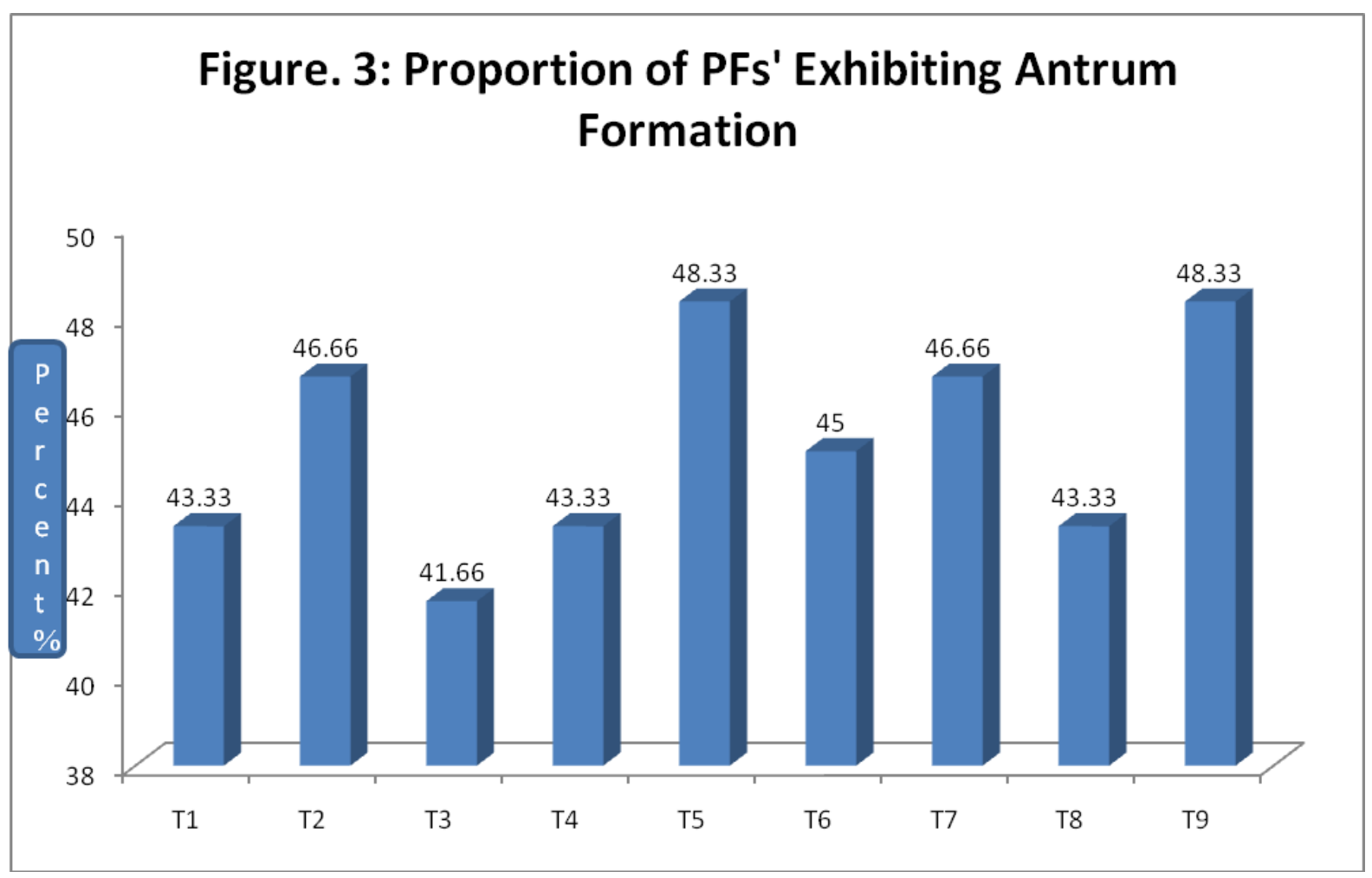

Figure.4: Proportion of Oocytes matured to MII stage

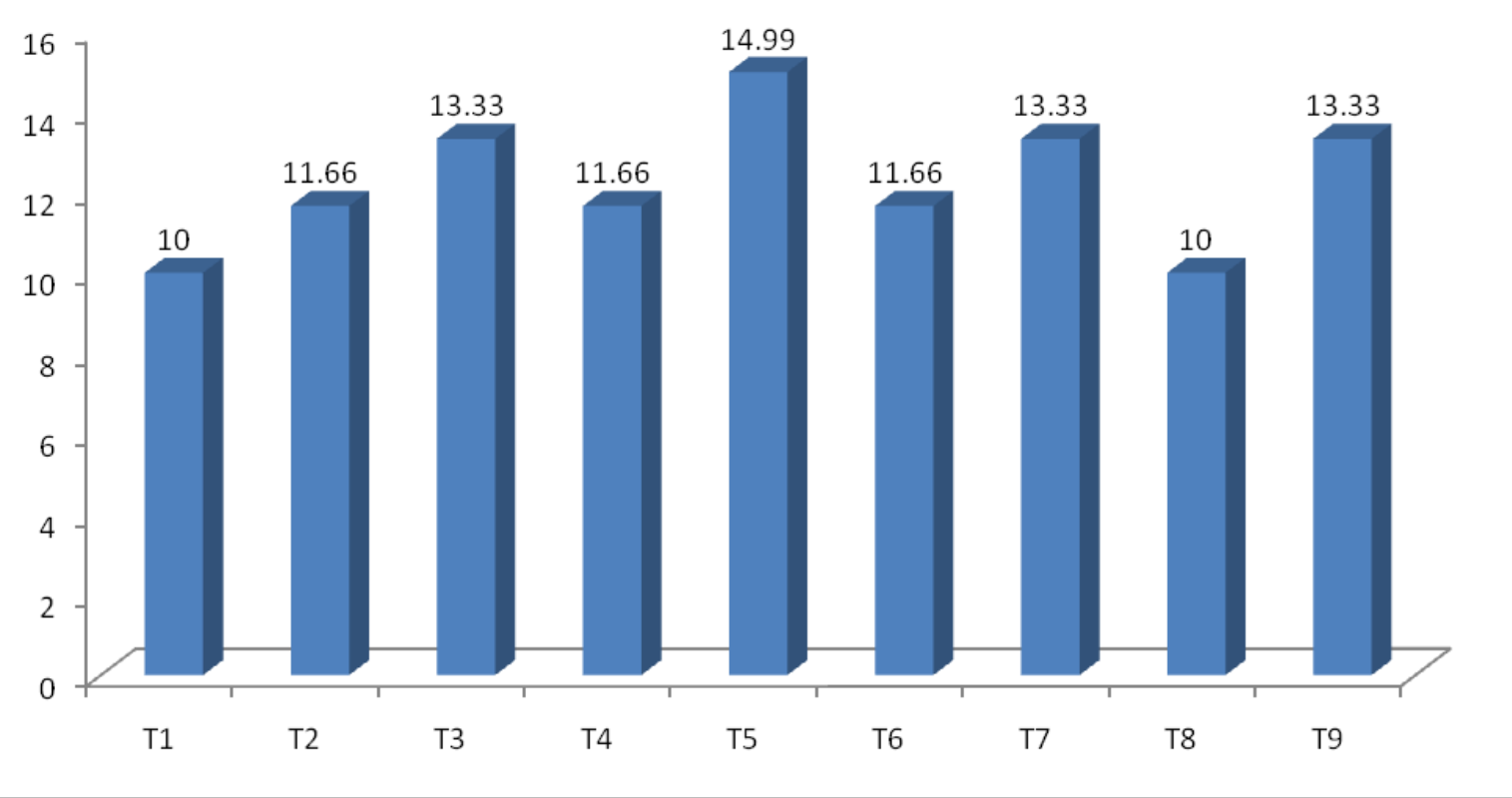


It was concluded that the supplementation of estradiol-17 $\beta$ during first two days of the culture supported better average increase in diameter and proportion of PFs' exhibiting antrum formation at the end of six day culture. Further the oocytes in COCs isolated at the end of culture in these treatments and subsequently subjected to in vitro maturation (IVM) for $24 \mathrm{hr}$ developed at a higher frequency to MII (metaphase II) stage.

Supplementation of estradiol-17 $\beta$ to TCM 199B culture medium in early stages followed by standard medium alone in later stages supports better development of PFs' in vitro. Following supplementation with estradiol-17 $\beta$ for the first two days culture of PFs' in standard medium appears to be advantageous for the development of preantral follicles in vitro.

\section{References}

Ali, A. and Sirard, M. A. 2002. The effects of 17 $\beta$-estradiol and protein supplement on the response to purified and recombinant follicle stimulating hormone in bovine oocytes. Zygote; 10: 65-71.

Araújo, V. R., Gastal, M. O., Figueiredo, J. $\mathrm{R}$ and Gastal, E. L. 2014. In vitro culture of bovine preantral follicles: a review. Reproductive Biology and Endocrinology ; 12:78.

Arunakumari, G., Shanmugasundaram, N. and Rao, V.H. 2010. Development of morulae from the oocytes of cultured sheep preantral follicles. Theriogenology; 74:884-894.

Beker, A. R. C. L., Colenbrander, B. and Bevers, M. M. 2002. Effect of $17 \beta-$ estradiol (E2) on the in vitro maturation of bovine oocytes. Theriogenology; 58:1663-1673.

Craig, Z. R., Singh, J., Gupta, R. K. and Flaws, J. A. 2014. Co-treatment of mouse antral follicles with $17 \beta$ estradiol interferes with mono-2ethyhexyl phthalate (MEHP)- induced atresia and altered apoptosis gene expression. Reproductive Toxicology; 45: 45-51.

Endo, M., Kawahara-Miki, R., Cao, F., Kimura, K., Kuwayama, T., Monji, $\mathrm{Y}$ and Iwata, H 2013. Estradiol supports in vitro development of bovine early antral follicles. Reproduction; 145: 85-96.

Li, Q., Niwa, K. and Hunter, M. G. 2004. Effect of $17 \beta$-estradiol on in vitro maturation of pig oocytes in proteinfree medium. Journal of Reproduction and Development; 50: 305-313.

Lima-Verde, I. B., Saraiva, M. V., Matos, M. H., Bruno, J. B., Tenório, S. B., Martins, F.S., Rossetto, R., Cunha, L.D, Name, K.P., Báo, S.N, Campello, C.C., and Figueiredo, J.R. 2010b. Interaction between estradiol and FSH promotes in vitro survival and development of caprine preantral follicles. Cells Tissues Organs; 191: 240-247.

Murdoch, W. J. 1998. Inhibition by oestrdiol of oxidative stress induced apoptosis in pig ovarian tissue. Journal of Reproduction and Fertility; 114: 127-130.

Nelson, L. R and Bulun, S. E. 2001. Estrogen production and action. Journal of American Acadamy Dermatology; 45: 116-124.

Tasaki, H., Iwata, H., Sato, D., Monji, Y. and Kuwayama, T. 2013. Estradiol has a major role in antrum formation of porcine preantral follicles cultured in vitro. Theriogenology; 79:809814.

Tesarik, K and Mendoza, C. 1995. Nongenemic effects of $17 \beta$-estradiol on maturing human oocytes: relations to oocytes developmental potential. Journal of Clinical Endocrinology and Metabolism; 80: 1438-1443. 
Tomic, D., Frech, M.S., Babus, J. K., Symonds, D., Furth, P. A., Koos, R. D and Flaws, J. A. 2007. Effect of ER $\alpha$ overexpression on female reproduction in mice. Reproduction Toxicology; 23: 317-325.
Zheng, P., Si, W., Bavister, B. D., Yang, J., Ding, C. and Ji, W. 2003. 17ß-estradiol and progesterone improve in vitro cytoplasmic maturation of oocytes from unstimulated prepubertal and adult rhesus monkeys. Human Reproduction; 18: 2137-2144.

\section{How to cite this article:}

Varaprasad Reddy. L.S.S, B. R. Naik, A. V. N. Sivakumar, B. Punyakumari and Suresh. J. 2020. In vitro Growth, Antrum Formation and Metaphase II Development of Preantral Follicles in Sheep with Estradiol-17 Supplementation at Different Time Points. Int.J.Curr.Microbiol.App.Sci. 9(01): 667-675. doi: https://doi.org/10.20546/ijcmas.2020.901.072 